\title{
Is alcohol consumption a risk factor for prostate cancer? A systematic review and meta-analysis
}

\author{
Jinhui Zhao ${ }^{1 *}$, Tim Stockwell ${ }^{1,2}$, Audra Roemer ${ }^{1,2}$ and Tanya Chikritzhs ${ }^{3}$
}

\begin{abstract}
Background: Research on a possible causal association between alcohol consumption and risk of prostate cancer is inconclusive. Recent studies on associations between alcohol consumption and other health outcomes suggest these are influenced by drinker misclassification errors and other study quality characteristics. The influence of these factors on estimates of the relationship between alcohol consumption and prostate cancer has not been previously investigated.
\end{abstract}

Methods: PubMed and Web of Science searches were made for case-control and cohort studies of alcohol consumption and prostate cancer morbidity and mortality (ICD-10: C61) up to December 2014. Studies were coded for drinker misclassification errors, quality of alcohol measures, extent of control for confounding and other study characteristics. Mixed models were used to estimate relative risk (RR) of morbidity or mortality from prostate cancer due to alcohol consumption with study level controls for selection bias and confounding.

Results: A total of 340 studies were identified of which 27 satisfied inclusion criteria providing 126 estimates for different alcohol exposures. Adjusted RR estimates indicated a significantly increased risk of prostate cancer among low $(\mathrm{RR}=1.08, P<0.001)$, medium $(\mathrm{RR}=1.07, P<0.01)$, high $(\mathrm{RR}=1.14, P<0.001)$ and higher $(\mathrm{RR}=1.18$, $P<0.001)$ volume drinkers compared to abstainers. There was a significant dose-response relationship for current drinkers $\left(P_{\text {trend }}<0.01\right)$. Studies free from misclassification errors produced the highest risk estimates for drinkers versus abstainers in adjusted models ( $R R=1.22, P<0.05$ ).

Conclusion: Our study finds, for the first time, a significant dose-response relationship between level of alcohol intake and risk of prostate cancer starting with low volume consumption $(>1.3,<24 \mathrm{~g}$ per day). This relationship is stronger in the relatively few studies free of former drinker misclassification error. Given the high prevalence of prostate cancer in the developed world, the public health implications of these findings are significant. Prostate cancer may need to be incorporated into future estimates of the burden of disease alongside other cancers (e.g. breast, oesophagus, colon, liver) and be integrated into public health strategies for reducing alcohol related disease.

Keywords: Prostate cancer, Alcohol, Meta-analysis, Misclassification error

\footnotetext{
*Correspondence: zhaoj@uvic.ca

${ }^{1}$ Centre for Addictions Research of British Columbia, University of Victoria, PO

Box 1700 STN CSC, Victoria, BC V8Y 2E4, Canada

Full list of author information is available at the end of the article
} 


\section{Background}

Prostate cancer is the development of cancer in the prostate, a walnut-sized gland in men that surrounds the top of the urethra and which produces seminal fluid [1]. Its growth and functions are controlled by male hormones such as testosterone. Prostate cancer is the second most common cancer in men worldwide. Around 1.1 million cases were recorded in 2012, accounting for $15 \%$ of all new cases of cancer in men [2]. It is most commonly diagnosed in high-income countries, where screening is common. It is the fifth most common cause of cancer death in men worldwide. Therefore prostate cancer as a chronic disease has become an important public health concern.

The risk factors for prostate cancer that can be considered established include age, race/ethnicity and family history [3]. Many observational studies have investigated alcohol consumption as a risk factor for prostate cancer. Conclusions from these studies and of reviews have been conflicting with some finding increased risk of prostate cancer [4-6], or decreased risk [7] and others finding no relationship [8-13]. While many unidentified and uncontrolled factors or biases may have confounded the relationships of interest in these studies, an additional concern is that former and occasional drinkers may be misclassified into the abstaining reference group. Previous studies have showed that such misclassification can bias estimates of health risks from alcohol use, for example, underestimating risks from low-volume drinking [14-19]. Former and occasional drinkers may include people who have stopped or reduced their drinking as they aged and experienced declining health $[16,20]$. Thus including former and occasional drinkers can bias the abstaining reference group towards reduced health and by comparison, reduce estimated disease risk from drinking.

Over the past few decades there have been several reviews and meta-analyses conducted to examine the association of prostate cancer with alcohol consumption [7, 8, 13, 21-25]. Early reviews by Longnecker [13] and Morton et al. [8] both concluded there was no relationship. Breslow and Weed [24] reviewed 32 studies of which only six reported significant associations between risk of prostate cancer and alcohol consumption. Dennis [22] conducted a meta-analysis on six cohort and 27 case-control studies, finding no overall association between prostate cancer and any alcohol consumption. However, when they examined 15 studies in which the relative risks (RR) for drinking levels were available, they found that three or more drinks per day increased the risk of prostate cancer. Dagnelie et al. (2004) [7] reviewed nine studies on prostate cancer and total alcohol consumption and found that six studies reported no association, two reported an increased risk and one a decreased risk. A meta-analysis by Bagnardi et al. [25] found a small but significantly increased risk for men drinking more than $50 \mathrm{~g} /$ day of alcohol, with a slightly higher risk for men consuming more than $100 \mathrm{~g} /$ day but there was no significant dose-response relation. This meta-analysis was the first to consider potential confounding, between-study variation and modifying effects of tobacco smoking but did not control for drinker misclassification errors. A meta-analysis by Fillmore et al. [21] found a significant relationship between prostate cancer and heavy alcohol use after controlling for the effects of median age of study populations, design and between-study variation. Rota et al. [23] found a significantly higher RR of prostate cancer for any drinking, light ( $\leq 1$ drink/day) and moderate drinking $(>1,<4$ drink/day) versus abstaining/occasional drinking but the analysis found no significant relationship with heavy drinking ( $\geq 4$ drinks/day) and did not consider the potential effects of misclassification. In summary, more recent reviews and meta-analyses have been more likely to find positive associations but none have adequately considered the effects of confounding and bias, including potential biases caused by misclassification of former and occasional drinkers in the abstainer reference groups.

The objectives of the present meta-analysis were: (i) to investigate the relationship between prostate cancer and alcohol consumption; and (ii) to examine whether estimates of this relationship may have been biased by drinker misclassification errors and other study characteristics.

\section{Methods}

\section{Inclusion and exclusion criteria}

The criteria for inclusion were: (i) case-control and cohort studies evaluating the relationship between alcohol consumption and prostate cancer; (ii) original articles published in English up till December 2014; (iii) articles that reported findings in odds ratio, hazard ratio, incidence ratio or standardized mortality ratio; and (iv) articles reporting at least three levels of alcohol consumption with drinking amounts, including the reference level. Articles with no abstainer group or a lowest drinking level greater than $0.33 \mathrm{~g} / \mathrm{d}$ were excluded. Additionally, studies reporting total alcohol consumption were included while studies based on consumption of specific beverages only such as wine, whiskey, vodka, sake or hard liquors were excluded. When the results of the study were published more than once or if the same dataset was used multiple times, only the most recent or more complete data were included in analyses. The primary outcomes of interest were mortality and/or morbidity from prostate cancer (ICD-9: 185 or ICD-10: C61) [26].

While published and peer reviewed cohort or casecontrol studies were included in the review, all other article types including narrative reviews, letters, editorials, 
commentaries, unpublished manuscripts, dissertations, government reports, books and book chapters, conference proceedings, meeting abstracts, lectures and address, and consensus development statement including guideline statements, were excluded.

\section{Search strategy}

The systematic review follows the Preferred Reporting Items for Systematic Reviews and Meta-Analyses (PRISMA) guidelines [27]. We identified all potentially relevant articles by searching Pubmed and Web of Science, through reference list cross-checking including those of previous meta-analyses and incorporating publications up to 31 December 2014. Hand searches of cited references in the selected articles, reviews and meta-analysis published on the same topic were also performed. The following MESH terms and text words were used: ("prostatic neoplasms" OR ("prostate" AND "neoplasms") OR "prostate cancer "OR ("prostate" AND "Cancer")) AND ("alcohol" OR (alcohol drinking) OR "alcohol consumption" OR "alcohol intake" OR ("alcohol" AND "consumption")).

\section{Study selection}

Two reviewers trained and supervised by the PI read the titles and/or abstracts of all the citations retrieved from the electronic database searches and removed all citations that were clearly not related to studies of the relationship between prostate cancer and alcohol consumption. The screening further involved abstract review. Full-text articles were obtained for all abstracts except for those that clearly did not meet eligibility criteria. The investigators were consulted in the event of any disagreement. Two of the investigators independently evaluated all studies selected for inclusion. The initial search identified a total of 340 studies of which 27 studies [4-6, 9, 11, 12, 28-48] satisfied the criteria for the meta-analysis after removing 313 records for reasons identified in Fig. 1.

\section{Data extraction}

Two reviewers independently reviewed all eligible papers to extract and code data from all studies fulfilling the inclusion criteria, and any disagreements were resolved by discussion with the investigators. Each study was coded with reference to a standardized code-book (available from authors on request) and under the supervision of investigators. The coding of all variables in the metadataset was double-checked by the first two authors. The data to be extracted were: (1) outcome, mortality or morbidity of prostate cancer; (2) measures of alcohol consumption; (3) study characteristics; (4) types of misclassification error of alcohol measure; and (5) controlled variables in individual studies.
A multitude of different approaches are used for assessing alcohol consumption in this literature [49]. Problematic approaches include assessing some beverage types and not others, assessing quantity consumed on a drinking day but not frequency, assessing consumption over very short time periods (e.g. two days) and assessing frequency but not quantity of consumption. We coded alcohol measurement as 'adequate' if both quantity and frequency of consumption was assessed for all alcoholic beverages and for a period of at least one week.

The primary exposure variable was level of daily alcohol consumption in grams of ethanol assessed at baseline and compared with a reference group of variously defined "non-drinkers" or "abstainers". When studies did not define the grams of alcohol per unit or drink, we used $8 \mathrm{~g} / \mathrm{unit}$ for the UK; $10 \mathrm{~g} /$ drink for Australia, Austria, France, Greece, Hungary, Ireland, Netherlands, New Zealand, Poland, Spain, Sweden; 11 g/drink for Finland; $12 \mathrm{~g} /$ drink for Denmark, Germany, Italy, South Africa and Switzerland; $13.45 \mathrm{~g} /$ drink for Canada; $14 \mathrm{~g} /$ drink for US; $12.5 \mathrm{~g} /$ drink for China, $19.75 \mathrm{~g} /$ drink for Japan and $12 \mathrm{~g} /$ drink for other countries [50, 51]. We converted alcohol intake into grams per day using the mid-points of reported categories to estimate mean values. Following practice in other meta-analyses involving self-reported alcohol consumption, the open-ended top categories (e.g. $6+$ drinks/day) were coded by adding three-quarters of the range of the next lowest category to the lower bound (e.g. if 3 to 5 drinks this would be 6 $+(5-3) * 0.75=7.5)$ [52]. It is necessary to make some higher estimate than the lowest level possible for these open-ended categories with no fixed upper level (e.g., 7.5 in this case instead of 6 for $6+$ drinks). We employed predetermined definitions of "low-volume" drinking (up to $20 \mathrm{~g}$ ethanol per day) based on Australian NHMRC low risk drinking guidelines [53]. This was operationalised as up to $24 \mathrm{~g}$ per day given that respondents in the studies reported whole drinks or units rather than grams i.e. $24 \mathrm{~g}$ per day is closer to two than three $10 \mathrm{~g}$ standard drinks per day. All data extracted from individual studies and analyzed during this study can be found in Additional file 1.

Studies were classified according to the presence or absence of two types of potential abstainer group bias: (i) including former drinkers and/or (ii) including occasional drinkers in the abstainer reference category. Studies were coded as having former drinker bias if a) results were not reported separately for former drinkers and b) there was no mention of removing former drinkers from the abstainer reference group. Following Fillmore et al. [16], lifetime abstention was strictly defined as zero consumption and did not include studies with any level of occasional lifetime or past year 


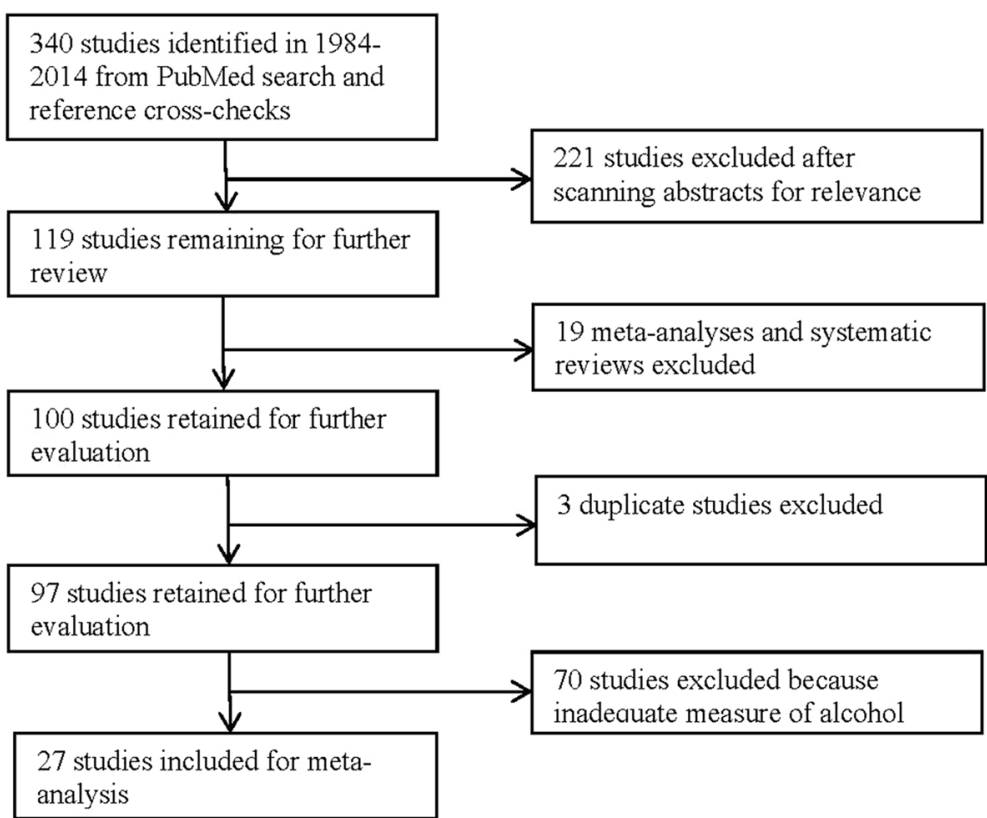

Fig. 1 Flowchart of summarizing systematic review of studies of prostate cancer morbidity or mortality and alcohol consumption from literature search to inclusion in meta-analysis

drinking (e.g. less than 12 drinks or "rarely" or "hardly ever" drinking). Our rationale for this strict criterion was that self-reported infrequent drinkers have been shown to greatly underreport their personal consumption $[54,55]$. Studies were coded as having occasional drinker bias if a) results were not reported separately for occasional drinkers and b) frequency of drinking was assessed for a "usual" period or over less than 30 days. The rationale here is that if a person reports "usually" not drinking over the course of a month, persons drinking less than monthly may still be occasional drinkers. When a study used occasional drinkers as the reference category and risk for abstainers was independently assessed, the risk values were recalculated using the abstainer category as the reference group [16].

\section{Strategy for data analysis}

Where studies only reported mortality or incidence rates, these were converted to RR estimates [56]. Otherwise hazard ratios in cohort studies and odds ratio estimates in case-control studies were entered as observations of the estimated risk relationships for meta-analysis. When the odds ratios (OR as RR estimates) are estimated using logistic regression models in a case-control study, the OR tends to overestimate RR when it is more than one and to underestimate RR when it is less than one if the outcome becomes more frequent [57]. Therefore, the formula below was used to correct the adjusted OR and its 95\% CIs obtained from logistic regression in studies and derive an estimate of an association that better represents the true RR [57].

$$
R R=\frac{O R}{\left(1-P_{0}\right)+\left(P_{0} \times O R\right)},
$$

where $R R$ is relative risk, $O R$ is odds ratio and $P_{0}$ is the incidence of outcome of interest in the non-exposed group.

Publication bias was assessed through visual inspection of the funnel plot of log-RR of morbidity or mortality of prostate cancer due to alcohol consumption against the inverse standard error of log-RR [56] and Egger's linear regression method [58]. We plotted a forest graph to examine how the RR estimate for any drinking in one study is different from others [56]. We also assessed between-study heterogeneity of RRs overall and by drinking groups using Cochran's Q [59] and the $\mathrm{I}^{2}$ statistic [60]. As no heterogeneity was detected, fixed effects models were used to obtain the summarized RR estimates [56]. We also conducted sensitivity tests using random effects models, but patterns of results were very similar and are not reported here.

We used the fixed effects models to estimate the weighted RRs of prostate cancer for any alcohol use and by drinking groups while adjusting for the potential effects of study-level covariates [56, 61-63]. Drinking level in each study group was examined in terms of predefined specific consumption levels. Drinking categories were defined and reclassified as: (1) lifetime occasional drinkers (0.02-0.33 g/day); (2) former drinkers now 
completely abstaining; (3) current occasional drinkers, up to one drink per week ( $<1.30 \mathrm{~g}$ per day); (4) low volume drinkers, up to 2 drinks or 1.30-24 g per day; (5) medium volume, up to 4 drinks or $25-44$ g per day; (6) high volume drinkers, up to 6 drinks or $25-64 \mathrm{~g}$ per day; and (7) higher volume drinkers, 6 drinks or $65 \mathrm{~g}$ or more per day. All studies had an open-ended heavier drinking group, i.e., with no upper limit of quantity consumed per day for responses accepted as valid. We investigated the dose-response relationship between the $R R$ and alcohol consumption for those who drank one drink or more per week using the midpoint of each exposure category using $t$-test in multivariate linear regression analysis [56].

We investigated the potential modification and confounding effects of study-level covariates using bivariate analysis of RR of prostate cancer morbidity or mortality and any alcohol consumption [64]. According to the availability of the data from 27 included studies, the following study characteristics were investigated: (1) study designs which included cohort study, population-based case-control study and hospital-based case-control study; (2) outcomes, i.e., morbidity or mortality of prostate cancer; (3) adequacy of drinking measurement method defined as whether both quantity and frequency of total alcohol consumption was assessed for at least one week; (4) mean or median age of individual study populations at baseline; (5) year at baseline, if recruited over a number of years then take midpoint; (6) whether subjects with a history of cancer were excluded at baseline or prior to randomization (yes, no or unknown); (7) presence of misclassification errors, i.e., including both former and occasional drinkers, only former drinkers, only occasional drinkers or neither former nor occasional drinkers in the abstaining reference group; (8) whether or not the study and control for social status (yes or no) using income or occupation measures; (9) whether or not a study controlled for racial identity or country of origin (yes or no); (10) whether or not a study control for smoking status (yes or no); (11) whether or not a study was conducted in US. We made stratified RR estimates for studies with different values for these characteristics and also examined the differences in the RR estimates between these same subgroups of studies [64].

The covariates above were selected for control in multivariate regression analyses on empirical grounds based on the $P$-value of bivariate tests of the log-RR of each covariate, and correlations with other covariates. Using all 27 studies, any variable whose bivariate test had a $P$-value $<0.10$ was considered as a candidate for the multivariate regression analyses of the $\log -\mathrm{RR}$ of prostate cancer morbidity or mortality $[65,66]$. If two or more covariates were moderately to highly correlated (coefficient $>0.30$ ), the one with lowest $P$-value from the bivariate test was included in the multivariate regression analyses. Abstainer bias was the main interest of the present study and thus its potential confounding effect was adjusted for in the pooled analysis (Table 3) and further examined in the stratified analysis (Table 4). On the basis of these criteria, two other covariates were included in the analyses: (i) whether or not the study was conducted in the US and (ii) whether smoking was controlled in the individual studies (Tables 3 and 4). Although the study design variable was not selected as a controlled covariate in the final models using bivariate analysis, the study design was a concern as these were unevenly distributed across the studies with different abstainer biases and the RR estimates were slightly different in case-control studies from cohort studies [23]. We still examined the potential effect of the design variable by performing a sensitivity analysis by including and excluding it in multivariate regression analyses (Tables 3 and 4). However, the estimates remained unchanged. We also conducted a correlation analysis of the study design variable and other selected covariates. The design variable was highly correlated with the abstainer bias variable (the coefficient $=0.48$ and $P<0.001$ ) and it was not included in the final models.

In multivariate regression analysis, the dependent variable was the natural log of the RR estimated using the rate ratio, hazard ratio or odds ratio of each drinking group in relation to the abstainer category. All analyses were weighted by the inverse of the estimated variance of the natural log RR. Variance was estimated from reported standard errors or confidence intervals. The weights for each individual study were created using the inverse variance weight scheme used in fixed regression analysis in order to obtain maximum precision for the main results of the meta-analysis [56] and such analyses may adjust for confounding among the characteristics [63].

Studies with large or small estimates and/or variance can be highly influential. Univariate analysis $[56,67,68]$ was performed to identify outliers. If a particular RR was more than twice the standard deviation of the RR estimates by drinking groups it was considered to be an outlier; five risk estimates were identified as outliers among 126 risk estimates. Sensitivity analyses were run after excluding outliers but no substantial changes in the risk estimates resulted [56]. A sensitivity analysis was also run after excluding one study by Putnam et al. [41] with markedly higher risk estimates but, again, the estimates remained unchanged. There was also no substantial effect on the RR estimates when each of other studies were excluded or included.

All significance tests assumed two-tailed $P$ values or 95\% CIs. All statistical analyses were performed using 
SAS 9.3 and the SAS PROC MIXED procedure was used to model the log-transformed RR [69].

\section{Role of the funding sources}

The study funders had no role in study design, data collection, analysis or interpretation, report preparation and the decision to publish. All authors had full access to all the data and had final responsibility for the decision to submit for publication.

\section{Results}

There were 126 risk estimates available for different alcohol exposures across the 27 selected studies. Table 1 presents the basic characteristics of these studies including covariates included in individual studies. As shown in Table 1, there were 16 prospective and one retrospective cohort studies, five hospital-based case-control and five population-based case-control studies. A forest plot (see Fig. 2) displays the weighted RR estimates for the risk of prostate cancer associated with any level of drinking versus "abstaining" reported in individual studies, grouped according to the type of misclassification error present. A visual inspection of Fig. 2 indicates considerable cross-study variation in estimates.

Table 2 presents unadjusted mean RR estimates of prostate cancer morbidity or mortality by level of alcohol consumption with tests of publication bias and heterogeneity. Figure 3 provides a funnel plot showing the $\log -R R s$ and their inverse standard error from which there was no indication of publication bias as the plot is reasonably symmetrical. No significant publication bias was detected using the Egger's regression either for the pooled data or the individual drinking categories data ( $P>0.05$ for each drinking category). Similarly, there was no significant heterogeneity detected using the $Q$ statistic in either the pooled or individual drinking category estimates $(P>0.05$ in each case). Compared to the "abstainers" (a heterogeneous group defined differently in different studies due to presence or absence of misclassification errors), being a drinker at any level was associated with increased risk of prostate cancer $(\mathrm{RR}=$ 1.08, 95\% CI: $1.04-1.12, P=0.0033)$. Risk of prostate cancer was significantly raised for low $(\mathrm{RR}=1.09, P=0.0031)$ and higher volume drinkers $(\mathrm{RR}=1.15, P=0.0336)$ but not other drinking categories. In unadjusted analysis, a significant dose-response relationship in the RR was observed among active drinkers (t-test statistic $=3.42, P=0.0009$ ).

We next examined whether study characteristics either significantly modified or potentially confounded the risk relationships between alcohol consumption and prostate cancer morbidity or mortality outcomes. The weighted RR estimate for any drinking versus non-drinking is significantly higher for US than non-US studies (t-test $P=$ 0.0005 ) but not significant for low volume drinking versus non-drinking (t-test $P=0.1432$ ) (see Additional file 2 . Weighted RR estimates according to study characteristics"). When further investigating whether the US vs. non-US variable was a modifier, the interaction term in the model was not statistically significant $(P=0.9580)$ and so meta-analyses are presented on a pool of both US and non-US studies. When tests with low volume alcohol exposure alone were conducted (see Additional file 2) a borderline modification effect with the misclassification error variable was evident $(P=0.0767)$ for the comparison between studies free of misclassification errors and those with just former drinker error. Two other variables were identified in bivariate analyses as potential confounders of the risk relationship between alcohol consumption and prostate cancer morbidity or mortality: (i) whether the USnon-US study $(P=0.0019)$ and (ii) whether a study controlled for smoking status $(P=0.0838)$. The misclassification error variable was included as covariates in the pooled (un-stratified) multivariate regression analysis given previous research highlighting their importance. Table 3 presents weighted only, partially adjusted and fully adjusted mean RR estimates of morbidity or mortality due to prostate cancer for different drinking categories. The weighted RR estimates without further adjustment were significantly higher for low, medium, high and higher volume drinkers than abstainers. After further adjusting for the confounding effect of drinker biases (partially adjusted), the RR estimates increased. After further adjusting for US-non-US study and controlled smoking (fully adjusted), there was a statistically significantly increased risk of prostate cancer for low (adjusted RR $=1.08,95 \% \mathrm{CI}=1.04-1.11$ and t-test $P=$ 0.0001), medium (adjusted $\mathrm{RR}=1.07,95 \% \mathrm{CI}=1.02-1.12$ and t-test $P=0.0041$ ), high (adjusted $\mathrm{RR}=1.14,95 \% \mathrm{CI}=$ 1.08-1.22 and t-test $P=0.0001$ ) and higher volume drinkers (adjusted $\mathrm{RR}=1.18,95 \% \mathrm{CI}=1.10-1.27$ and $\mathrm{t}-$ test $P=0.0001)$. There was also still a significant dose-response relation between risk of prostate cancer and alcohol consumption for current drinkers in adjusted analysis (Fully adjusted model, $\mathrm{t}$-test statistic $=2.79, \mathrm{P}_{\text {trend }}=0.0063$ ). Figure 4 presents the adjusted RRs for different drinking levels.

Given the previous literature indicating the potential for misclassification errors to bias risk estimates, visual inspection of the Fig. 2 and the borderline evidence for effect modification in Additional file 2, we also present results stratified by type of misclassification errors detected in Table 4 . These show substantially different estimates according to the presence or absence of different misclassification errors with studies free from errors having the highest RR estimate for low volume drinkers $(\mathrm{RR}=1.23,95 \% \mathrm{CI}: 1.05-1.45, P=0.0143)$ and those with only former drinker bias having the lowest $(R R=$ 1.01, 95\% CI: $0.96-1.06, P=0.6901$ ). A similar pattern of results was evident for higher levels of alcohol 
Table 1 Characteristics of 27 included studies for meta-analysis on prostate cancer and alcohol consumption

\begin{tabular}{|c|c|c|c|c|c|c|c|}
\hline Author & Study country & Cases $/ N^{a}$ & Outcome $^{\text {b }}$ & Design $^{c}$ & Age range & Follow-up yrs & Covariates assess $^{d}$ \\
\hline \multicolumn{8}{|l|}{ Studies with both biases } \\
\hline Stemmermann, [11] & US & $227 / 8006$ & $M / M$ & P-cohort & $46-65$ & 25.5 & 1,4 \\
\hline Tavani et al., [12] & Italy & $281 / 880$ & Morb & Hos-CC & $25-79$ & $\mathrm{u} / \mathrm{a}$ & $1,2,4,5$ \\
\hline Breslow et al., [30] & US & $252 / 5766$ & $\mathrm{M} / \mathrm{M}$ & P-cohort & $25-75$ & 17.0 & $1,2,3$ \\
\hline Schuurman et al., [44] & Netherlands & $680 / 58279$ & Morb & P-cohort & $55-69$ & 6.3 & 1,2 \\
\hline Lund Nilsen et, [37] & Norway & $644 / 22895$ & $M / M$ & P-cohort & $40-99$ & 9.3 & 1 \\
\hline Ellison, [34] & Canada & $145 / 3400$ & $M / M$ & R-cohort & $50-84$ & 23.0 & 1 \\
\hline Sesso et al., [4] & US & $366 / 7612$ & $M / M$ & P-cohort & $30-68$ & 5.0 & $1,4,5,6$ \\
\hline Velicer et al., [46] & US & $816 / 34565$ & Morb & P-cohort & $50-76$ & 4.0 & 1 \\
\hline McGregor et al., [38] & Canada & $947 / 1986$ & Morb & Pop-CC & -79 & $\mathrm{u} / \mathrm{a}$ & 1 \\
\hline Sawada et al., [43] & Japan & $913 / 48218$ & Morb & P-cohort & $40-79$ & 16.0 & $1,4,5$ \\
\hline \multicolumn{8}{|c|}{ Studies with former drinker bias only } \\
\hline Jain et al., [35] & Canada & $617 / 1254$ & Morb & Pop-CC & $48-92$ & $\mathrm{u} / \mathrm{a}$ & 1,4 \\
\hline Putnam et al., [41] & US & $101 / 1572$ & $\mathrm{M} / \mathrm{M}$ & P-cohort & $40-86$ & 9.0 & 1 \\
\hline Platz et al., [40] & US & $2479 / 47843$ & $\mathrm{M} / \mathrm{M}$ & P-cohort & $40-75$ & 12.0 & $1,3,5,6$ \\
\hline Weinstein et al., [47] & Finland & $1270 / 27111$ & $\mathrm{M} / \mathrm{M}$ & P-cohort & $50-69$ & 17.0 & 1,7 \\
\hline Rohrmann et al., [42] & Europe $^{\mathrm{e}}$ & $2655 / 142647$ & $M / M$ & P-cohort & $40-65$ & 8.7 & 4,5 \\
\hline Watters et al., [5] & US & $17227 / 294707$ & $\mathrm{M} / \mathrm{M}$ & P-cohort & $50-71$ & 7.0 & $1,2,3,4,5,6$ \\
\hline \multicolumn{8}{|c|}{ Studies with occasional drinker bias only } \\
\hline Hiatt et al., [9] & US & $238 / 43432$ & Morb & P-cohort & $30-99$ & 4.6 & $1,2,3$ \\
\hline Andersson et al., [28] & Sweden & $256 / 508$ & Morb & Pop-CC & -79 & $\mathrm{u} / \mathrm{a}$ & 1 \\
\hline Hayes et al., [6] & US & $981 / 2296$ & Morb & Pop-CC & $40-79$ & $\mathrm{u} / \mathrm{a}$ & 1,3 \\
\hline Baglietto et al., [29] & Australia & $732 / 16872$ & $M / M$ & P-cohort & $27-70$ & 10.3 & 1 \\
\hline Breslow et al., [48] & US & $8362 / 323354$ & Mort & P-cohort & 18-99 & 8.3 & $1,2,3,5$ \\
\hline \multicolumn{8}{|c|}{ Studies with neither abstainer bias } \\
\hline De Stefani et al., [33] & Uruguay & $156 / 458$ & Morb & Hos-CC & $40-89$ & $\mathrm{u} / \mathrm{a}$ & $1,2,4$ \\
\hline Lumey et al., [36] & US & $699 / 2740$ & Morb & Hos-CC & $36-81$ & $\mathrm{u} / \mathrm{a}$ & $1,2,3$ \\
\hline Crispo et al., [32] & Italy & $2663 / 4114$ & Morb & Hos-CC & $46-74$ & $\mathrm{u} / \mathrm{a}$ & $1,2,5,6$ \\
\hline Chang et al., [31] & Sweden & $1499 / 2629$ & Morb & Pop-CC & $45-79$ & $\mathrm{u} / \mathrm{a}$ & 1 \\
\hline Pelucchi et al., [39] & Italy & $1294 / 2745$ & Morb & Hos-CC & $46-79$ & $\mathrm{u} / \mathrm{a}$ & 1 \\
\hline Sutcliffe et al., [45] & US & $3348 / 45433$ & $M / M$ & P-cohort & $40-75$ & 16.0 & $1,3,5,6$ \\
\hline
\end{tabular}

Note: ${ }^{\mathrm{a}} \mathrm{N}=$ cases + controls in a case-control study. ${ }^{\mathrm{b}} \mathrm{M} / \mathrm{M}=$ mortality and morbidity, Morb $=$ morbidity and Mort $=$ mortality. ${ }^{\mathrm{c}} \mathrm{P}-$ cohort $=$ prospective cohort, $\mathrm{R}$-cohort $=$ retrospective cohort, Pop-CC = population-based case-control, Hos-CC = hospital-based case-control. ${ }^{\mathrm{d}} 1$ : age; 2 : social status; 3 : race; 4: smoking status; 5: body mass index; 6: exercise. ${ }^{\mathrm{e}} 10$ European countries: Denmark, France, Germany, Great Britain, Greece, Italy, The Netherlands, Norway, Spain and Sweden

consumption and, also, for estimates of prostate cancer risk with any level of current alcohol consumption for which only the error-free studies show a significant risk for drinking regardless of whether adjustment is made whether studies controlled for US-non-US study or smoking. Sensitivity analysis found that inclusion or exclusion of the study design variable in the models made no difference to the estimates. These results are basically consistent with the pooled analysis in suggesting an increased risk even for low volume drinking but also indicate the importance of misclassification errors as a potential cause of bias. In particular, inclusion of former drinkers in the abstaining reference group appears to reduce the risk estimates.

\section{Discussion}

Meta-analyses of cohort and case-control studies were conducted to investigate (i) the role of alcohol consumption as a potential risk factor for prostate cancer and, (ii) whether this relationship was significantly influenced by key study characteristics and potential biases, in particular according to whether former and/or occasional drinkers were misclassified as abstainers. Unique among published meta-analyses [21-23, 25], we report a significant 


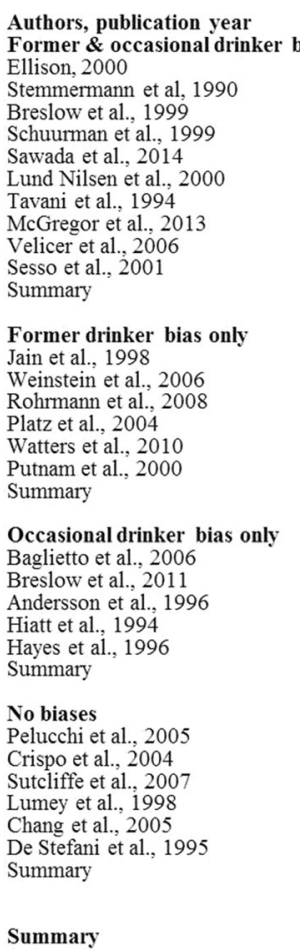

Summary

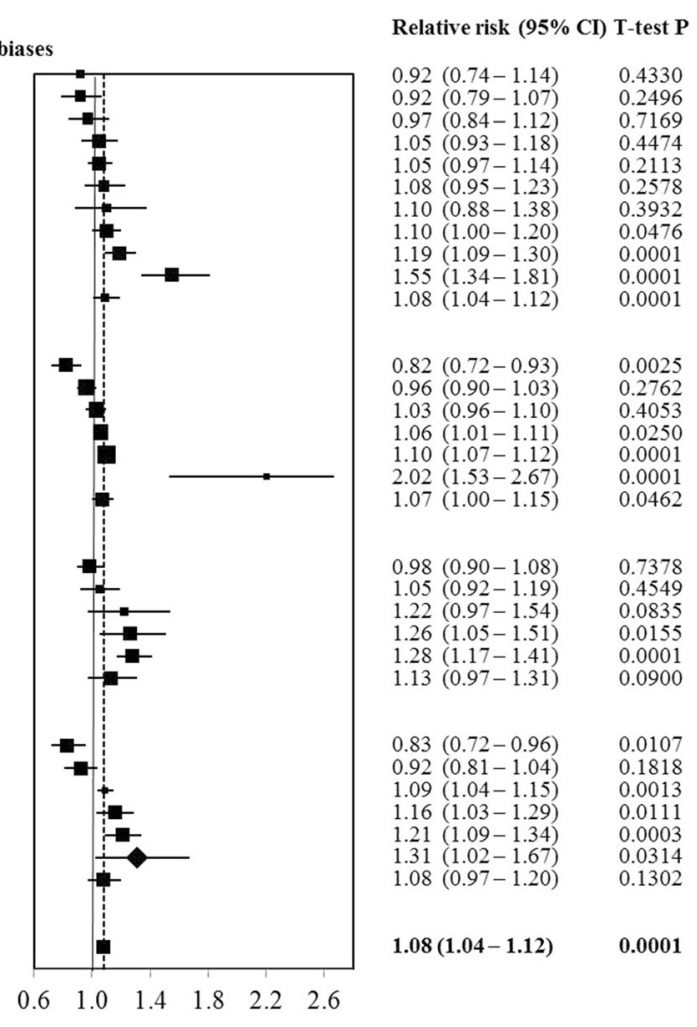

Relative risk (95\% CI)

Fig. 2 Relative risk $(95 \% \mathrm{Cl})$ of prostate cancer morbidity or mortality for any alcohol consumption versus "abstaining" in 27 studies

dose response relationship to be observed with increasing risk of prostate cancer starting at low-level alcohol consumption $(>1.33 \mathrm{~g}$ and $<25 \mathrm{~g}$ ethanol/day) regardless of adjustment for study characteristics in pooled models of all 27 eligible studies. High (45- $<65$ g/day) and higher $(65+\mathrm{g} /$ day) volume drinkers had a significantly higher risk ( $R R=$ 1.14 and 1.18). Further, there was no significant heterogeneity in study estimates or evidence of publication bias. However, when analyses were stratified by whether or not studies misclassified former and/or occasional drinkers as abstainers, it was evident that former drinker bias reduced overall risk estimates to the extent that alcohol exposure at any level was no longer associated with significantly increased risk of prostate cancer. Out of 27 studies included, 16 contained former drinker bias, 15 occasional drinker bias only, six were free from both types of bias. It can be concluded that the common practice of combining former drinkers with abstainers in prospective studies of alcohol

Table 2 Unadjusted mean RR estimates of prostate cancer morbidity or mortality for different categories of drinkers compared with 'abstainers' ( $N=27$ studies and 126 observations) with tests of publication bias and heterogeneity

\begin{tabular}{|c|c|c|c|c|c|c|c|}
\hline \multirow[t]{2}{*}{ Drinking categories } & \multirow[t]{2}{*}{$N / n^{a}$} & \multicolumn{2}{|c|}{ Unadjusted mean RR } & \multicolumn{2}{|c|}{ Egger's regression for publication bias } & \multicolumn{2}{|c|}{ Test for heterogeneity } \\
\hline & & $\mathrm{RR}(95 \% \mathrm{Cl})$ & $\overline{t \text {-test } P}$ & Coefficient & $t$-test $P$ & Q statistic $P$ & $\mathrm{I}^{2}(\%, 95 \% \mathrm{Cl})$ \\
\hline Abstainer & & 1.00 & & & & & \\
\hline Former drinker & $7 / 13$ & $1.04(0.92-1.19)$ & 0.5155 & -1.33 & 0.2274 & $>0.05$ & $1.00(0.00-56.59)$ \\
\hline Occasional (<1.30 g/day) & $6 / 7$ & $1.02(0.86-1.21)$ & 0.8292 & +1.63 & 0.0542 & $>0.05$ & $1.00(0.00-70.81)$ \\
\hline Low volume $(1.30-<25$ g/day $)$ & $27 / 62$ & $1.09(1.03-1.16)$ & 0.0031 & +0.13 & 0.5558 & $>0.05$ & $10.66(0.00-64.80)$ \\
\hline Medium volume (25- < 45 g/day) & $18 / 20$ & $1.03(0.93-1.14)$ & 0.6046 & -0.26 & 0.4287 & $>0.05$ & $1.00(0.00-62.37)$ \\
\hline High volume (45- < 65 g/day) & $10 / 11$ & $1.13(0.98-1.30)$ & 0.0935 & -0.32 & 0.5125 & $>0.05$ & $13.38(0.00-53.90)$ \\
\hline Higher volume (65+ g/day) & $9 / 13$ & $1.15(1.01-1.13)$ & 0.0336 & -0.24 & 0.6353 & $>0.05$ & $19.94(0.00-57.85)$ \\
\hline Any drinking & $27 / 126$ & $1.08(1.04-1.12)$ & 0.0033 & -0.04 & 0.7727 & $>0.05$ & $16.42(0.00-33.73)$ \\
\hline
\end{tabular}




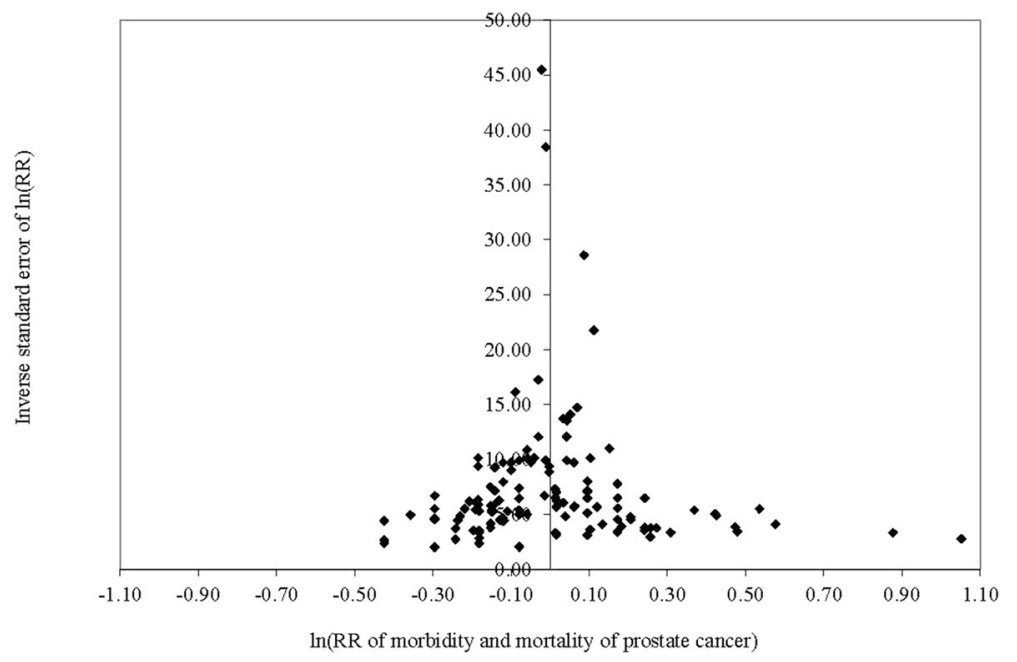

Fig. 3 Funnel plot of relative risk (In(RR)) of prostate cancer morbidity or mortality due to alcohol consumption against inverse standard error of In (RR)

consumption and health biases risk estimates downwards and can lead to underestimation of the risks posed by low volume consumption. There was no indication that misclassifying occasional drinkers contributed to significant downward bias in risk estimates and, further, when estimates were made separately for occasional drinkers the RRs tended to be slightly lower. We conclude that the common practice of misclassifying former drinkers as abstainers, especially in older studies, has sometimes disguised a significant association between alcohol exposure and risk of prostate cancer.

Alcohol is a known carcinogen causing a variety of human cancers [70] via different biological pathways depending on the anatomical site. The evidence that alcoholic drinks are a cause of cancers of the mouth, pharynx, larynx, oesophagus, liver, colorectum and breast in women is compelling [25, 70, 71]. Alcoholic beverages are multicomponent mixtures containing several carcinogenic compounds such as ethanol, acetaldehyde, aflatoxins and ethyl carbamate [72] and all of these compounds may contribute to increase the risk of cancer due to alcohol consumption reported in observational studies. The biological mechanisms by which alcohol intake might increase the risk of prostate cancer are not fully understood but the main mechanisms are likely to include a genotoxic effect of acetaldehyde, the induction of microsomal cytochrome P450 2E1 (CYP2E1) and associated oxidative stress, increased estrogen concentration, a role as a solvent for tobacco carcinogens, changes in folate metabolism, and changes in DNA repair [73-75].

Several limitations with our meta-analysis must be acknowledged. Our meta-analysis was based on 27 studies including 126 risk estimates. This sample is relatively small when conducting multivariate regression to control for study level characteristics that might confound

Table 3 Adjusted mean RR estimates of prostate cancer morbidity or mortality for different categories of drinkers compared with abstainers ( $N=27$ studies and 126 risk estimates)

\begin{tabular}{|c|c|c|c|c|c|c|c|c|c|c|}
\hline \multirow[t]{2}{*}{ Drinking categories } & \multirow[t]{2}{*}{$\mathrm{N} / \mathrm{n}^{\mathrm{a}}$} & \multicolumn{3}{|c|}{ Weighted Mean RR ${ }^{b}$} & \multicolumn{3}{|c|}{ Partially adjusted mean $R R^{c}$} & \multicolumn{3}{|c|}{ Fully adjusted mean $R^{d}$} \\
\hline & & \multicolumn{2}{|c|}{ RR \& $95 \% \mathrm{Cl}$} & \multirow[t]{2}{*}{$t$-test $P$} & \multicolumn{2}{|c|}{ RR \& $95 \% \mathrm{Cl}$} & \multirow[t]{2}{*}{$t$-test $P$} & \multicolumn{2}{|c|}{ RR \& $95 \% \mathrm{Cl}$} & \multirow[t]{2}{*}{$t$-test $P$} \\
\hline Abstainer & & 1.00 & & & 1.00 & & & 1.00 & & \\
\hline Former drinker & $7 / 13$ & 1.14 & $0.99-1.30$ & 0.0642 & 1.12 & $0.98-1.29$ & 0.0990 & 1.10 & $0.97-1.25$ & 0.1348 \\
\hline Occasional (<1.30 g/day) & $6 / 7$ & 0.97 & $0.86-1.10$ & 0.6126 & 0.96 & $0.85-1.08$ & 0.5265 & 0.95 & $0.85-1.06$ & 0.3263 \\
\hline Low volume $(1.30-<25 \mathrm{~g} /$ day $)$ & $27 / 62$ & 1.07 & $1.04-1.10$ & 0.0001 & 1.08 & $1.05-1.12$ & 0.0001 & 1.08 & $1.04-1.11$ & 0.0001 \\
\hline Medium volume ( $25-<45$ g/day) & $18 / 20$ & 1.06 & $1.02-1.11$ & 0.0068 & 1.08 & $1.03-1.13$ & 0.0023 & 1.07 & $1.02-1.12$ & 0.0041 \\
\hline High volume $(45-<65$ g/day) & $10 / 11$ & 1.15 & $1.08-1.22$ & 0.0001 & 1.17 & $1.09-1.25$ & 0.0001 & 1.14 & $1.08-1.22$ & 0.0001 \\
\hline Higher volume $(65+\mathrm{g} /$ day $)$ & $9 / 13$ & 1.18 & $1.09-1.27$ & 0.0001 & 1.20 & $1.11-1.29$ & 0.0001 & 1.18 & $1.10-1.27$ & 0.0001 \\
\hline Any drinking & $27 / 126$ & 1.08 & $1.04-1.12$ & 0.0039 & 1.10 & $1.02-1.18$ & 0.0214 & 1.08 & $1.01-1.17$ & 0.0364 \\
\hline
\end{tabular}

Note: ${ }^{a} N=$ Number of studies and $n=$ Number of risk estimates. ${ }^{b}$ Weighted using the inverse of variance of natural log-RR. ${ }^{c}$ Weighted RR estimates adjusted for both former and occasional drinker biases. ${ }^{d}$ Weighted RR estimates adjusted for between-study variation, both former and occasional drinker biases, US/non-US study and control for smoking status in individual studies 


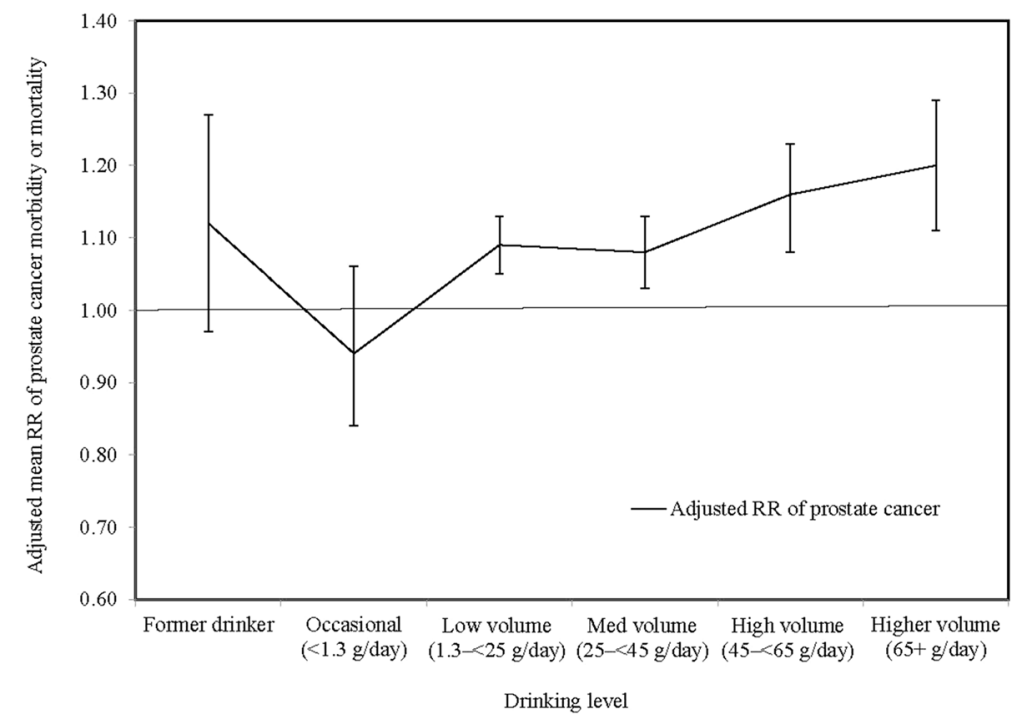

Fig. 4 Adjusted mean relative risk (RR) of prostate cancer morbidity or mortality due to alcohol consumption

the relationship between prostate cancer and alcohol consumption. Furthermore, adjustment for study level characteristics such as whether smoking status was controlled is of course not as precise as controlling for this variable at the individual level within a study. Inevitably, uncontrolled confounding from unmeasured or imprecisely measured variables will be present both within and between studies. Control for smoking status, for example, can be done in many ways and some studies did not distinguish former smokers from lifetime nonsmokers. Our analysis showed a statistically significantly higher risk of prostate cancer due to any drinking in the studies conducted in the US than in other countries. However, this effect disappeared when controls for other

Table 4 Adjusted mean RR estimates of prostate cancer morbidity or mortality for different categories of drinkers compared with 'abstainers' by misclassification errors

\begin{tabular}{|c|c|c|c|c|c|c|c|}
\hline \multirow[t]{2}{*}{ Drinking categories } & \multirow[t]{2}{*}{$\mathrm{N} / \mathrm{n}^{\mathrm{a}}$} & \multicolumn{3}{|c|}{ Weighted for mean RR } & \multicolumn{3}{|c|}{ Adjusted for mean $R^{b}$} \\
\hline & & $\overline{\mathrm{RR}}$ & $95 \% \mathrm{Cl}$ & $\overline{t \text {-test } P}$ & $\overline{R R}$ & $95 \% \mathrm{Cl}$ & $t$-test $P$ \\
\hline \multicolumn{8}{|l|}{ Former \& occasional drinker biases } \\
\hline Low-volume $(1.30-<25 \mathrm{~g} /$ day $)$ & $10 / 20$ & 1.10 & $1.03-1.18$ & 0.1499 & 1.11 & $1.03-1.19$ & 0.0069 \\
\hline Medium-high volume (25+ g/day) & $9 / 17$ & 1.12 & $1.02-1.22$ & 0.0152 & 1.13 & $1.04-1.24$ & 0.0079 \\
\hline Any drinking $(1.30+\mathrm{g} /$ day $)$ & $10 / 37$ & 1.11 & $1.05-1.17$ & 0.0273 & 1.12 & $1.02-1.23$ & 0.0405 \\
\hline \multicolumn{8}{|l|}{ Former drinker bias only } \\
\hline Low-volume $(1.30-<25 \mathrm{~g} /$ day $)$ & $6 / 16$ & 1.05 & $1.00-1.10$ & 0.0708 & 1.01 & $0.96-1.06$ & 0.6901 \\
\hline Medium-high volume (25+ g/day) & $5 / 10$ & 1.10 & $1.05-1.16$ & 0.0005 & 1.05 & $0.99-1.12$ & 0.1136 \\
\hline Any drinking (1.30+ g/day) & $6 / 26$ & 1.07 & $0.84-1.37$ & 0.1657 & 1.03 & $0.87-1.22$ & 0.2760 \\
\hline \multicolumn{8}{|l|}{ Occasional drinker bias only } \\
\hline Low-volume $(1.30-<25$ g/day $)$ & $5 / 10$ & 1.08 & $0.97-1.21$ & 0.1411 & 1.06 & $0.95-1.18$ & 0.2534 \\
\hline Medium-high volume (25+ g/day) & $4 / 9$ & 1.06 & $0.95-1.18$ & 0.2674 & 1.01 & $0.89-1.15$ & 0.8706 \\
\hline Any drinking (1.30+ g/day) & $5 / 19$ & 1.07 & $0.97-1.18$ & 0.0715 & 1.04 & $0.83-1.29$ & 0.2955 \\
\hline \multicolumn{8}{|l|}{ Neither former or occasional drinker biases } \\
\hline Low-volume $(1.30-<25$ g/day $)$ & $6 / 16$ & 1.12 & $1.05-1.18$ & 0.0008 & 1.23 & $1.05-1.45$ & 0.0143 \\
\hline Medium-high volume (25+ g/day) & $3 / 8$ & 1.10 & $0.95-1.26$ & 0.1798 & 1.20 & $1.00-1.43$ & 0.0475 \\
\hline Any drinking (1.30+ g/day) & $6 / 24$ & 1.11 & $1.03-1.19$ & 0.0359 & 1.22 & $1.07-1.38$ & 0.0321 \\
\hline
\end{tabular}


covariates such as drinker bias and between-study variation were introduced. This finding might be affected by the relatively small number of studies available for analysis and/or other unmeasured confounders and modifiers. The issue should be revisited in future metaanalyses when more studies are available. While there were relatively few studies available for analysis, the required number of subjects per variable for linear regression is much smaller than in logistic regression and a minimum of even two subjects per variable would not bias the estimate in linear regression analysis [76]. The great majority of identified studies suffered one or more serious methodological problems including the widespread practice of misclassifying former drinkers as abstainers. Our study was unable to incorporate the recommendation from Liang et al. [77] that former drinkers should in fact be included within the category of current drinkers according to previous drinking level so as to create an unbiased estimate of the risk relationship as only two eligible studies provided the risk estimates of differential drinking levels among former drinkers $[6,36]$. Finally, as highlighted by Zeisser et al. [19], many studies classified occasional drinkers as low or medium volume drinkers creating the possibility of "reverse occasional drinker bias". This may have differential effects according to gender but in the present study male occasional drinkers mostly had the lowest level of risk of prostate cancer. If occasional drinkers are included with low volume drinkers this could also have the effect of minimizing risk estimates. No studies were identified that were free of all possible types of misclassification error (ie when reverse occasional drinker bias is considered).

\section{Conclusions}

In summary, the RR of prostate cancer morbidity or mortality significantly increased at low volume alcohol consumption levels ( $>1.3 \mathrm{~g}$ and $<24 \mathrm{~g}$ per day) compared to abstinence and a statistically significant a dose-response relationship was observed for the first time in a meta-analysis. The level of increased risk observed for low volume drinkers was relatively modest in the pooled analysis (8\%), but was as high as $23 \%$ in the studies free of misclassification error. In either scenario, with a condition as prevalent as prostate cancer in developed countries, the public health implications of the findings are significant, so we suggest these are practically significant levels of risk for prevention purposes. Different forms of misclassification bias may differentially affect risk estimates, particularly the common practice of including former drinkers in the abstainer reference group may reduce risk estimates. Prostate cancer may need to be incorporated in future estimates of the burden of disease alongside other cancers (e.g. breast, oesophagus, colon, liver) and be integrated into public health strategies for reducing alcohol related disease. We recommend that future prospective studies on alcohol and disease seek to avoid biasing risk estimates by misclassifying either (i) former or occasional drinkers as abstainers, or (ii) occasional drinkers as low volume drinkers. Following Liang and Chikritzhs [77], we also recommend that former drinkers are classified with drinkers according to their past level of consumption.

\section{Additional files}

Additional file 1: Meta-analysis data on prostate cancer and alcohol consumption. (XLSX $42 \mathrm{~kb}$ )

Additional file 2: Weighted RR estimates according to study characteristics. (DOCX 44 kb)

\section{Acknowledgement}

We thank the authors of included studies who provided the data necessary for our meta-analysis. We thank Sapna Panwar who initially conducted the systematic searches working with Tanya Chikritzhs and Tim Stockwell. Tanya Chikritzhs and Tim Stockwell received fees from the Institute for Scientific Analysis, San Francisco, USA for some of their contributions during the course of the wider research program this study is a part of. We also thank Drs Alan Bostrom from the Institute for Scientific Analysis, San Francisco and Scott Macdonald, University of Victoria for valuable comments on an earlier draft of this paper.

\section{Funding}

This study was funded by the US National Institutes of Health [Award \#1RO1AAO19939-02].

\section{Availability of data and materials}

All data generated and analyzed during this study are included in this published article and can be found in Additional file 1: Meta-analysis data on prostate cancer and alcohol consumption.

\section{Authors' contribution}

TC (PI) and TS (co-investigator) were involved in the study design of the project "Systematic error and confounding: Meta-analyses of alcohol and disease" funded by the NIH [Award \#1RO1AAO19939-02]. TS, TC, AR and JZ were involved in literature search, study selection, data extraction. JZ, TS and AR checked the data. JZ and TS conducted the statistical analyses,

interpreted the results and wrote the first draft of the original manuscript. TC revised the manuscript and all authors approved the final manuscript.

\section{Competing interests}

JZ, TC and AR declare no competing interests. TS declares funding received from Systembolaget, the Swedish retail alcohol monopoly for a study of public health impacts of restrictive alcohol policies. Systembolaget reports to the Swedish Ministry of Health and has a mandate to limit the public health consequences of alcohol consumption.

\section{Consent for publication \\ Not applicable.}

Ethics approval and consent to participate Not applicable.

\section{Author details}

${ }^{1}$ Centre for Addictions Research of British Columbia, University of Victoria, PO Box 1700 STN CSC, Victoria, BC V8Y 2E4, Canada. ${ }^{2}$ Department of Psychology, University of Victoria, PO Box 1700 STN CSC, Victoria, BC V8Y 2E4, Canada. ${ }^{3}$ National Drug Research Institute, Curtin University, GPO box U1987, Perth 6845, WA, Australia.

Received: 24 August 2015 Accepted: 25 October 2016 Published online: 15 November 2016 


\section{References}

1. Bostwick DG, Crawford DE, Higano CS, Roach III M. American Cancer Society's complete guide to prostate cancer. Atlanta: American Cancer Society; 2004.

2. Forman D, Ferlay J. The global and regional burden of cancer. In: Wild BWSaCP, editor. World Cancer Report 2014. Lyon: International Agency for Research on Cancer (IARC); 2014. p. 16-53.

3. Gann PH. Risk factors for prostate cancer. Rev Urol. 2002;4 Suppl 5:S3-S10.

4. Sesso HD, Paffenbarger RS, Lee IM. Alcohol consumption and risk of prostate cancer: The Harvard Alumni Health Study. Int J Epidemiol. 2001;30(4):749-55.

5. Watters JL, Park Y, Hollenbeck A, Schatzkin A, Albanes D. Alcoholic Beverages and Prostate Cancer in a Prospective US Cohort Study. Am J Epidemiol. 2010;172(7):773-80.

6. Hayes RB, Brown LM, Schoenberg JB, Greenberg RS, Silverman DT, Schwartz AG Swanson GM, Benichou J, Liff JM, Hoover RN, et al. Alcohol use and prostate cancer risk in US blacks and whites. Am J Epidemiol. 1996;143(7):692-7.

7. Dagnelie PC, Schuurman AG, Goldbohm RA, Van den Brandt PA. Diet, anthropometric measures and prostate cancer risk: a review of prospective cohort and intervention studies. BJU Int. 2004;93(8):1139-50.

8. Morton MS, Griffiths K, Blacklock N. The preventive role of diet in prostatic disease. Brit J Urol. 1996;77(4):481-93.

9. Hiatt RA, Armstrong MA, Klatsky AL, Sidney S. Alcohol-Consumption, Smoking, and Other Risk-Factors and Prostate-Cancer in a Large Health Plan Cohort in California (United-States). Cancer Cause Control. 1994;5(1):66-72.

10. Vandergulden JWJ, Verbeek ALM, Kolk JJ. Smoking and Drinking Habits in Relation to Prostate-Cancer. Brit J Urol. 1994;73(4):382-9.

11. Stemmermann GN, Nomura AMY, Chyou PH, Yoshizawa C. Prospective-Study of Alcohol Intake and Large-Bowel Cancer. Digest Dis Sci. 1990;35(11):1414-20.

12. Tavani A, Negri E, Franceschi S, Talamini R, Lavecchia C. AlcoholConsumption and Risk of Prostate-Cancer. Nutr Cancer. 1994;21(1):25-31.

13. Longnecker MP. Alcohol-Consumption and Risk of Cancer in Humans - an Overview. Alcohol. 1995;12(2):87-96.

14. Stockwell T, Greer A, Fillmore K, Chikritzhs T, Zeisser C. Health Benefits of Moderate Alcohol Consumption How Good Is the Science? Brit Med J. 2012;344.

15. Chikritzhs T, Fillmore K, Stockwell T. A healthy dose of scepticism: Four good reasons to think again about protective effects of alcohol on coronary heart disease. Drug Alcohol Rev. 2009;28(4):441-4.

16. Fillmore KM, Kerr WC, Stockwell T, Chikritzhs T, Bostrom A. Moderate alcohol use and reduced mortality risk: Systematic error in prospective studies. Addict Res Theory. 2006;14(2):101-32.

17. Fillmore KM, Stockwell T, Chikritzhs T, Bostrom A, Kerr W. Moderate alcohol use and reduced mortality risk: Systematic error in prospective studies and new hypotheses. Ann Epidemiol. 2007;17(5):S16-23.

18. Roizen R, Fillmore K, Chikritzhs T, Stockwell T. Light-to-moderate drinking and dementia risk: The former drinkers problem re-visited. Addict Res Theory. 2013;21(3):181-93.

19. Zeisser C, Stockwell TR, Chikritzhs T. Methodological Biases in Estimating the Relationship Between Alcohol Consumption and Breast Cancer: The Role of Drinker Misclassification Errors in Meta-Analytic Results. Alcohol Clin Exp Res. 2014;38(8):2297-306

20. Shaper AG, Wannamethee G, Walker M. Alcohol and mortality in British men: explaining the U-shaped curve. Lancet. 1988:2(8623):1267-73.

21. Fillmore KM, Chikritzhs T, Stockwell T, Bostrom A, Pascal R. Alcohol use and prostate cancer: A meta-analysis. Mol Nutr Food Res. 2009:53(2):240-55.

22. Dennis LK. Meta-analysis for combining relative risks of alcohol consumption and prostate cancer. Prostate. 2000:42(1):56-66.

23. Rota M, Scotti L, Turati F, Tramacere I, Islami F, Bellocco R, Negri E, Corrao G, Boffetta P, La Vecchia C, et al. Alcohol consumption and prostate cancer risk: a meta-analysis of the dose-risk relation. Eur J Cancer Prev. 2012;21(4):350-9.

24. Breslow RA, Weed DL. Review of epidemiologic studies of alcohol and prostate cancer: 1971-1996. Nutr Cancer. 1998;30(1):1-13.

25. Bagnardi V, Blangiardo M, La Vecchia C, Corrao G. A meta-analysis of alcohol drinking and cancer risk. Brit J Cancer. 2001;85(11):1700-5.

26. WHO. International statistical classification of diseases and related health problems. Geneva: WHO; 2010. Available from URL: http://www.who.int/ classifications/icd/ICD10Volume2_en_2010.pdf. Accessible 3 Oct 2013.

27. Moher D, Liberati A, Tetzlaff J, Altman DG, Grp P. Preferred Reporting Items for Systematic Reviews and Meta-Analyses: The PRISMA Statement. Plos Med. 2009;6(7).

28. Andersson SO, Baron J, Bergstrom R, Lindgren C, Wolk A, Adami HO Lifestyle factors and prostate cancer risk: A case-control study in Sweden. Cancer Epidem Biomar. 1996;5(7):509-13.
29. Baglietto L, Severi G, English DR, Hopper JL, Giles GG. Alcohol consumption and prostate cancer risk: Results from the Melbourne collaborative cohort study. Int J Cancer. 2006;119(6):1501-4.

30. Breslow RA, Wideroff L, Graubard BI, Erwin D, Reichman ME, Ziegler RG, Ballard-Barbash R. Alcohol and prostate cancer in the NHANES I epidemiologic follow-up study. Ann Epidemiol. 1999;9(4):254-61.

31. Chang ET, Hedelin M, Adami HO, Gronberg H, Balter KA. Alcohol drinking and risk of localized versus advanced and sporadic versus familial prostate cancer in Sweden. Cancer Cause Control. 2005;16(3):275-84.

32. Crispo A, Talamini R, Gallus S, Negri E, Gallo A, Bosetti C, La Vecchia C, Dal Maso L, Montella M. Alcohol and the risk of prostate cancer and benign prostatic hyperplasia. Urology. 2004;64(4):717-22.

33. DeStefani E, Fierro L, Barrios E, Ronco A. Tobacco, alcohol, diet and risk of prostate cancer. Tumori. 1995;81(5):315-20.

34. Ellison LF. Tea and other beverage consumption and prostate cancer risk: a Canadian retrospective cohort study. Eur J Cancer Prev. 2000;9(2):125-30.

35. Jain MG, Hislop GT, Howe GR, Burch JD, Ghadirian P. Alcohol and other beverage use and prostate cancer risk among Canadian men. Int J Cancer. 1998;78(6):707-11.

36. Lumey LH, Pittman B, Wynder EL. Alcohol use and prostate cancer in US whites: No association in a confirmatory study. Prostate. 1998;36(4):250-5.

37. Nilsen $T I L$, Johnsen R, Vatten $L$. Socio-economic and lifestyle factors associated with the risk of prostate cancer. Brit J Cancer. 2000;82(7):1358-63.

38. McGregor SE, Courneya KS, Kopciuk KA, Tosevski C, Friedenreich CM. Casecontrol study of lifetime alcohol intake and prostate cancer risk. Cancer Cause Control. 2013;24(3):451-61.

39. Pelucchi C, Galeone C, Talamini R, Negri E, Parpinel M, Franceschi S, Montella M, La Vecchia C. Dietary folate and risk of prostate cancer in Italy. Cancer Epidem Biomar. 2005;14(4):944-8.

40. Platz EA, Leitzmann MF, Rimm EB, Willett WC, Giovannucci E. Alcohol intake, drinking patterns, and risk of prostate cancer in a large prospective cohort study. Am J Epidemiol. 2004;159(5):444-53.

41. Putnam SD, Cerhan JR, Parker AS, Bianchi GD, Wallace RB, Cantor KP, Lynch CF. Lifestyle and anthropometric risk factors for prostate cancer in a cohort of lowa men. Ann Epidemiol. 2000;10(6):361-9.

42. Rohrmann S, Linseisen J, Key TJ, Jensen MK, Overvad K, Johnsen NF, Tjonneland A, Kaaks R, Bergmann MM, Weikert C, et al. Alcohol consumption and the risk for prostate cancer in the European prospective investigation into cancer and nutrition. Cancer Epidem Biomar. 2008;17(5):1282-7.

43. Sawada N, Inoue M, Iwasaki M, Sasazuki S, Yamaji T, Shimazu T, Tsugane S. Alcohol and smoking and subsequent risk of prostate cancer in Japanese men: The Japan Public Health Center-based prospective study. Int J Cancer. 2014;134(4):971-8

44. Schuurman AG, Goldbohm RA, van den Brandt PA. A prospective cohort study on consumption of alcoholic beverages in relation to prostate cancer incidence (The Netherlands). Cancer Cause Control. 1999;10(6):597-605.

45. Sutcliffe S, Giovannucci E, Leitzmann MF, Rimm EB, Stampfer MJ, Willett WC, Platz EA. A prospective cohort study of red wine consumption and risk of prostate cancer. Int J Cancer. 2007;120(7):1529-35.

46. Velicer CM, Kristal A, White E. Alcohol use and the risk of prostate cancer: Results from the VITAL cohort study. Nutr Cancer. 2006;56(1):50-56

47. Weinstein SJ, Stolzenberg-Solomon R, Pietinen P, Taylor PR, Virtamo J, Albanes D. Dietary factors of one-carbon metabolism and prostate cancer risk. Am J Clin Nutr. 2006;84(4):929-35.

48. Breslow RA, Chen CM, Graubard BI, Mukamal KJ. Prospective Study of Alcohol Consumption Quantity and Frequency and Cancer-Specific Mortality in the US Population. Am J Epidemiol. 2011;174(9):1044-53.

49. Stockwell T, Chikritzhs T. Commentary: Another serious challenge to the hypothesis that moderate drinking is good for health? Int J Epidemiol. 2013:42(6):1792-4.

50. Tumer C. How much alcohol is in a 'standard drink'? An analysis of 125 studies. Brit J Addict. 1990;85(9):6.

51. International Alliance for Responsible Drinking. Drinking Guidelines: General Population. International Alliance for Responsible Drinking; 2016. Available from URL: http://www.iard.org/policy-tables/drinking-guidelines-generalpopulation/. Accessed 29 Oct 2016.

52. Roerecke M, Rehm J. The cardioprotective association of average alcohol consumption and ischaemic heart disease: a systematic review and metaanalysis. Addiction. 2012;107(7):1246-60.

53. National Health and Medical Research Council of Australia. Australian guidelines to reduce health risk from drinking alcohol. Canberra: AttorneyGeneral's Department; 2009. 
54. Stockwell T, Zhao JH, Macdonald S. Who under-reports their alcohol consumption in telephone surveys and by how much? An application of the 'yesterday method' in a national Canadian substance use survey. Addiction. 2014;109(10):1657-66.

55. Ye Y, Bond J, Cherpitel CJ, Stockwell T, Macdonald S, Rehm J. Risk of Injury Due to Alcohol Evaluating Potential Bias Using the Case-Crossover UsualFrequency Method. Epidemiology. 2013;24(2):240-3.

56. Woodward M. Epidemiology study design and data analysis. Boca Raton: Chapman \& Hall/CRC; 2000.

57. Zhang J, Yu KF. What's the relative risk? A method of correcting the odds ratio in cohort studies of common outcomes. Jama-J Am Med Assoc. 1998;280(19):1690-1.

58. Egger M, Smith GD, Schneider M, Minder C. Bias in meta-analysis detected by a simple, graphical test. Brit Med J. 1997;315(7109):629-34.

59. Cochran WG. The Combination of Estimates from Different Experiments. Biometrics. 1954;10(1):101-29.

60. Higgins JPT, Thompson SG. Quantifying heterogeneity in a meta-analysis. Stat Med. 2002;21(11):1539-58.

61. Borenstein M, Hedges LV, Higgins JPT, Rothstein HR. Meta-regression. In: Borenstein M, Hedges LV, Higgins JPT, Rothstein HR, editors. Introduction to meta-analysis. West Sussex: John Wiley \& Sons, Ltd; 2009. p. 127.

62. van Houwelingen $H C$, Arends LR, Stijnen T. Advanced methods in metaanalysis: multivariate approach and meta-regression. Stat Med. 2002;21(4): 589-624.

63. Greenland S. Meta-analysis. In: Rothman KJ, Greenland S, editors. Modern epidemiology. Philadelphia: Lippincott Williams \& Wilkins; 1998. p. 32.

64. Greenland S, Rothman K. Introduction to stratified analysis. In: Rothman KJ, Greenland S, editors. Modern Epidemiology. Philadelphia: Lippincott Williams \& Wilkins; 1998. p. 28.

65. Hosmer DW, Lemeshow S. Applied logistic regression. New York: Wiley; 2000.

66. Dales LG, Ury HK. Improper Use of Statistical Significance Testing in Studying Covariables. Int J Epidemiol. 1978;7(4):373-5.

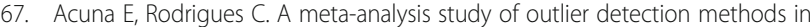
classification. Mayaguez: University of Puerto Rico at Mayaguez; 2014. Available from URL: http://academic.uprm.edu/eacuna/paperout.pdf. Accessible 4 Sept 2014

68. Pagano M, Gauvreau K. Principles of biostatistics. 2nd ed. Duxbury: Pacific Grove, CA; 2000.

69. Institute SAS. SAS/STAT 9.3 user's guide. Cary: SAS Institute Inc.; 2011.

70. World Cancer Research Fund/American Institute for Cancer Research. Food, nutrition, physical activity, and the prevention of cancer: A global perspective. Washington DC: AICR; 2007.

71. English DR, Holman C, D'Arcy J, Australia Department of Community Services and Health National Drug Strategy. The quantification of drug caused morbidity and mortality in Australia/prepared for the Department of Human Services and Health for use in association with the National Drug Strategy. Canberra: Australia Government Publication Service; 1995.

72. Lachenmeier DW, Przybylski MC, Rehm J. Comparative risk assessment of carcinogens in alcoholic beverages using the margin of exposure approach. Int J Cancer. 2012;131(6):E995-E1003.

73. IARC. IARC monographs on the evaluation of carcinogenic risks to humans: alcohol consumption and ethyl carbamate, vol. 96. Lyon: IARC; 2010.

74. Baan R, Straif K, Grosse Y, Secretan B, El Ghissassi F, Bouvard V, Altieri A, Cogliano V, Monograph WIARC. Carcinogenicity of alcoholic beverages. Lancet Oncol. 2007:8(4):292-3.

75. Homann N, Stickel F, Konig IR, Jacobs A, Junghanns K, Benesova M, Schuppan D, Himsel S, Zuber-Jerger I, Hellerbrand C, et al. Alcohol dehydrogenase $1 C^{*} 1$ allele is a genetic marker for alcohol-associated cancer in heavy drinkers. Int J Cancer. 2006;118(8):1998-2002.

76. Austin PC, Steyerberg EW. The number of subjects per variable required in linear regression analyses. J Clin Epidemiol. 2015;68(6):627-36.

77. Liang WB, Chikritzhs T. The Association between Alcohol Exposure and SelfReported Health Status: The Effect of Separating Former and Current Drinkers. Plos One. 2013:8(2).

\section{Submit your next manuscript to BioMed Central and we will help you at every step:}

- We accept pre-submission inquiries

- Our selector tool helps you to find the most relevant journal

- We provide round the clock customer support

- Convenient online submission

- Thorough peer review

- Inclusion in PubMed and all major indexing services

- Maximum visibility for your research

Submit your manuscript at www.biomedcentral.com/submit

) Biomed Central 